\title{
Prevalência de hipertensão pulmonar avaliada por ecocardiografia Doppler em uma população de pacientes adolescentes e adultos com fibrose cística*
}

\author{
Prevalence of pulmonary hypertension evaluated by Doppler echocardiography \\ in a population of adolescent and adult patients with cystic fibrosis \\ Paula Maria Eidt Rovedder ${ }^{1}$, Bruna Ziegler $^{2}$, Antônio Fernando Furlan Pinotti ${ }^{3}$, \\ Sérgio Saldanha Menna Barreto ${ }^{4}$, Paulo de Tarso Roth Dalcin ${ }^{5}$
}

\begin{abstract}
Resumo
Objetivo: Determinar a prevalência de hipertensão pulmonar (HP) em pacientes com fibrose cística (FC), comparar características clínicas, escore radiológico, função pulmonar e parâmetros ecocardiográficos nos grupos com e sem HP e correlacionar achados ecocardiográficos com características clínicas, escore radiológico e função pulmonar. Métodos: Estudo transversal prospectivo em pacientes clinicamente estáveis (idade $\geq 16$ anos) atendidos por um programa de adultos para FC. Os pacientes foram submetidos a avaliação clínica, ecocardiografia Doppler, testes de função pulmonar e exame radiológico do tórax. Resultados: Obteve-se a velocidade de regurgitação tricúspide (VRT) em 37 dos 40 pacientes estudados. A prevalência de HP foi de 49\% com um ponte de corte da VRT de 2,5 m/s (18 pacientes) e de $30 \%$ com um ponte de corte da VRT de $2,8 \mathrm{~m} / \mathrm{s}$ (11 pacientes). Os valores de saturação periférica de oxigênio $\left(\mathrm{SpO}_{2}\right)$ em repouso, escore clínico, volume expiratório forçado no primeiro segundo $\left(\mathrm{VEF}_{1}\right)$ e capacidade vital forçada (CVF) foram significativamente menores no grupo com HP. A VRT correlacionou-se significativamente com $\mathrm{SpO}_{2}$ em repouso $(\mathrm{p}<0,001)$, escore clínico $(\mathrm{p}<0,001)$, escore radiológico $(\mathrm{p}=0,030)$, VEF $\mathrm{em}$ litros $(p<0,001)$ e em \% do previsto $(p<0,001)$ e CVF em litros $(p=0,008)$ e em \% do previsto $(p=0,001)$. A SpO $\mathrm{S}_{2}$ em repouso foi o melhor preditor independente da VRT $(p<0,001)$. Conclusão: A alta prevalência de HP nos pacientes com FC estudados sugere que a presença de HP seja considerada na avaliação e acompanhamento desses pacientes. 0 melhor preditor de $\mathrm{HP}$ foi a $\mathrm{SpO}_{2}$ em repouso.
\end{abstract}

Descritores: Fibrose cística; Valva Tricúspide/fisiopatologia; Hipertensão pulmonar; Ecocardiografia Doppler.

\begin{abstract}
Objective: To determine the prevalence of pulmonary hypertension (PH) in patients with cystic fibrosis (CF), to compare clinical characteristics, radiographic scores, pulmonary function, and echocardiographic parameters in patients with and without $\mathrm{PH}$, and to correlate echocardiographic findings with clinical characteristics, radiographic scores, and pulmonary function. Methods: This was a prospective, cross-sectional study involving clinically stable patients (aged 16 or older) enrolled in an adult CF program. The patients were submitted to clinical evaluation, Doppler echocardiography, pulmonary function tests, and chest X-rays. Results: Tricuspid regurgitant jet velocity (TRV) was obtained in 37 of the 40 patients studied. The prevalence of $\mathrm{PH}$ was $49 \%$ with a TRV cut-off of $2.5 \mathrm{~m} / \mathrm{s}$ (18 patients) and $30 \%$ with a TRV cut-off of $2.8 \mathrm{~m} / \mathrm{s}$ (11 patients). Peripheral oxygen saturation $\left(\mathrm{SpO}_{2}\right)$ at rest, clinical score, forced expiratory volume in one second $\left(\mathrm{FEV}_{1}\right)$, and forced vital capacity (FVC) were significantly lower in the group with $\mathrm{PH}$. The TRV was found to correlate significantly with Sp0 at rest $(p<0.001)$, clinical score $(p<0.001)$, radiographic score $(p=0.030)$, FEV in liters $(p<0.001)$ and in $\%$ of predicted $(p<0.001)$, and FCV in liters $(p=0.008)$ and in $\%$ of predicted $(p=0.001)$. The single best predictor of TRV was SpO at rest $(p<0.001)$. Conclusion: The high prevalence of $\mathrm{PH}$ in the $\mathrm{CF}$ patients studied suggests that $\mathrm{PH}$ should be considered in the evaluation and follow-up treatment of such patients. The best predictor of $\mathrm{PH}$ was $\mathrm{SpO}_{2}$ at rest.
\end{abstract}

Keywords: Cystic fibrosis; Tricuspid valve/physiopathology; Hypertension, pulmonary; Echocardiography, Doppler.

\footnotetext{
* Trabalho realizado no Serviço de Pneumologia do Hospital de Clínicas de Porto Alegre. Universidade Federal do Rio Grande do Sul - UFRGS - Porto Alegre (RS) Brasil. 1. Professora Assistente da Faculdade de Fisioterapia. Centro Universitário Metodista Instituto Porto Alegre - IPA - Porto Alegre (RS) Brasil.

2. Fisioterapeuta do Serviço de Pneumologia. Hospital de Clínicas de Porto Alegre. Universidade Federal do Rio Grande do Sul - UFRGS - Porto Alegre (RS) Brasil. 3. Cardiologista Ecocardiografista do Serviço de Cardiologia. Hospital de Clínicas de Porto Alegre, Porto Alegre (RS) Brasil.

4. Professor Titular do Departamento de Medicina Interna. Faculdade de Medicina da Universidade Federal do Rio Grande do Sul - UFRGS - Porto Alegre (RS) Brasil. 5. Professor Adjunto do Departamento de Medicina Interna. Faculdade de Medicina da Universidade Federal do Rio Grande do Sul - UFRGS - Porto Alegre (RS) Brasil. Endereço para correspondência: Paula Maria Eidt Rovedder. Rua Domingos Crescêncio, 185/502, Bairro Santana, CEP 90650-090, Porto Alegre, RS, Brasil.

Tel 5551 3210-8241. E-mail: larove_@hotmail.com

Recebido para publicação em 21/1/2007. Aprovado, após revisão, em 13/6/2007.
} 


\section{Introdução}

A fibrose cística $(\mathrm{FC})$ é uma doença genética autossômica recessiva de evolução crônica, progressiva e irreversível. É identificada clinicamente pela tríade: doença pulmonar obstrutiva supurativa crônica, insuficiência pancreática e níveis elevados de eletrólitos no suor. ${ }^{(1,2)}$

Embora a FC tenha caráter multissistêmico, o acometimento pulmonar é o determinante principal de morbidade e mortalidade nessa afecção. Com o avançar da doença pulmonar, uma grande porcentagem dos pacientes com FC desenvolve hipertensão pulmonar (HP). Nesses pacientes o desenvolvimento de hipóxia alveolar (em áreas hipoventiladas) conduz à vasoconstrição pulmonar hipóxica. Quando o estado hipóxico é prolongado, a circulação pulmonar sofre alterações estruturais chamadas de remodelamento, ou seja, ocorre hipertrofia e hiperplasia da camada média dos vasos arteriais, bem como muscularização de vasos periféricos. ${ }^{(3-5)}$

0 cateterismo cardíaco é o exame diagnóstico mais preciso para determinar o surgimento de HP, e a técnica não-invasiva mais sensivel para o reconhecimento precoce e monitoramento da HP é a ecocardiografia Doppler. (4,5) $^{(1,}$

A ecocardiografia permite verificar a existência da HP, quantificá-la, verificar sua variabilidade e verificar as repercussões para câmaras direitas, assim como permite avaliações seriadas após intervenções terapêuticas. Achados freqüentes na avaliação de pacientes com HP incluem dilatação das cavidades direitas, hipertrofia do ventrículo direito, movimento paradoxal do septo e insuficiência tricúspide. ${ }^{(4,6,7)}$

De acordo com alguns estudos, ${ }^{(8,9)}$ existe uma grande porcentagem de pacientes com FC que apresentam HP subclínica. Dessa maneira, a grande variabilidade fenotípica da doença( ${ }^{(10)}$ justificaria uma abordagem diagnóstica sistemática para avaliação de HP em uma população de pacientes adolescentes e adultos de nosso meio. Além disso, a possibilidade de que parâmetros quantitativos da rotina clínica, como escore clínico, escore radiológico, saturação periférica de oxigênio $\left(\mathrm{SpO}_{2}\right)$ e variáveis espirométricas, possam indicar o momento de rastrear a presença de HP nesses pacientes também motiva a realização de um novo estudo sobre o tema.

0 objetivo deste estudo foi, então, determinar a prevalência de hipertensão pulmonar, por meio da ecocardiografia Doppler, em pacientes atendidos por um programa de adultos para FC, comparar características clínicas, escore radiológico, função pulmonar e parâmetros ecocardiográficos nos grupos classificados como com e sem HP e correlacionar indicadores de função ventricular direita com características clínicas, escore radiológico e função pulmonar.

\section{Métodos}

A população do estudo constituiu-se de pacientes com diagnóstico de FC, de acordo com critérios de consenso, ${ }^{(11)}$ atendidos pelo programa de adultos do Hospital de Clínicas de Porto Alegre (HCPA). Os pacientes deveriam ter idade $\geq 16$ anos e estar clinicamente estáveis. 0 critério de exclusão do estudo foi a presença de doença cardíaca primária.

Este estudo teve caráter transversal e prospectivo. Inicialmente, cada paciente foi submetido a uma avaliação clínica para definir a estabilidade clínica e o escore clínico da doença. Em seguida, dentro de um período de uma semana, os pacientes foram submetidos a um estudo ecocardiográfico com Doppler, aos testes de função pulmonar e a um exame radiológico convencional do tórax.

Todos os pacientes foram submetidos a um ecocardiograma transtorácico bidimensional modo-M com Doppler (ATL-HDI 5000 Philips, Bothell, WA, EUA). 0 estudo ecocardiográfico foi realizado, mediante a padronização das janelas paraesternal, apical e subcostal com o paciente em repouso, por um único observador cegado para o estado clínico do paciente. As medidas ecocardiográficas foram executadas de acordo com as recomendações da Sociedade Americana de Cardiologia. ${ }^{(12)}$ A regurgitação de tricúspide foi avaliada pelo refluxo sangüíneo através da válvula tricúspide, em cada contração do ventrículo direito, mediante corte apical paraesternal das quatro câmaras. O pico de velocidade de regurgitação tricúspide (VRT) foi usado para estimar o gradiente sistólico da pressão com o uso da equação modificada de Bernoulli:

$$
\Delta p=4 v^{2}
$$

onde $\Delta p$ corresponde à pressão delta, e $v$ corresponde à velocidade. Para fins de análise, a HP foi definida como uma VRT $>2,5 \mathrm{~m} / \mathrm{s}$. Também se utilizou um ponto de corte mais conservador para a VRT, 2,8 m/s, para determinar a prevalência de HP. 
0 tempo de aceleração sistólica (TAS) do ventrículo direito/artéria pulmonar foi calculado, mediante traçado de fluxo anterógrado pulmonar, como o intervalo entre o início do fluxo na artéria pulmonar e a velocidade do pico de fluxo. 0 diâmetro do ventrículo direito (DVD) foi medida no final da diástole.

A determinação das dimensões das câmaras esquerdas foi obtida pelo modo-M, através da janela paraesternal. A fração de ejeção foi avaliada pelo método de Teichholz. A massa ventricular esquerda foi calculada por meio da fórmula modificada de Devereux, corrigida pela área de superfície corporal e expressa também como índice de massa. 0 diâmetro do átrio esquerdo foi determinado, no final da ejeção ventricular esquerda, medindo-se a distância vertical da face interna da parede posterior da aorta à parede posterior do átrio.

0 escore de avaliação clínica utilizado foi o de Shwachman-Kulczycki. ${ }^{(13)}$ Esse sistema de avaliação clínica considera quatro diferentes características (atividade geral, exame físico, nutrição e achados radiológicos do tórax), sendo cada uma delas pontuada em uma escala de 5 a 25 pontos.

0 exame radiológico convencional do tórax foi realizado em todos os indivíduos incluídos no estudo. Um único profissional sênior da área de Pneumologia, o qual estava cegado para as condições clínicas e a identificação dos pacientes, realizou a pontuação do escore radiológico utilizando o sistema de escore radiológico de Brasfield et al. ${ }^{(14)}$ Foram assinaladas pontuações, em grau crescente de gravidade, para cada uma das seguintes características: alçaponamento de ar ( 0 a 4), marcas lineares ( 0 a 4), lesões cístico-nodulares ( 0 a 4 ), lesões extensas do espaço aéreo ( 0,3 ou 5) e gravidade geral ( 0 a 5). 0 escore total: 25 menos o total de pontos assinalados nas 5 características examinadas.

Os testes de função pulmonar foram realizados com espirômetro computadorizado (Jaeger-v4.31, Jaeger, Würzburg, Alemanha). Foram realizadas três tentativas, sendo a melhor delas registrada. Os parâmetros estudados foram capacidade vital forçada (CVF) em litros e em porcentagem do previsto e volume expiratório forçado no primeiro segundo $\left(\mathrm{VEF}_{1}\right)$ em litros e em porcentagem do previsto para idade, estatura e gênero. ${ }^{(15)}$ Os fluxos aéreos foram analisados de acordo com as Diretrizes para Testes de Função Pulmonar da Sociedade Brasileira de Pneumologia e Tisiologia.
A avaliação da saturação periférica de oxigênio $\left(\mathrm{SpO}_{2}\right)$ foi realizada com o paciente em repouso utilizando-se o oxímetro de pulso modelo NPB-40 (Nellcor Puritan Bennett; Pleasanton, CA, EUA).

Os dados foram digitados em base de dados no programa Microsoft Excel 2000, sendo analisados com auxílio do programa Statistical Package for the Social Sciences, versão 13,0. Os dados quantitativos foram apresentados como média \pm desvio padrão ou como mediana (intervalo interquartílico). Os dados qualitativos foram expressos em $\mathrm{n}$ (\% de todos os casos).

Os dados quantitativos com distribuição normal foram analisados pelo teste $t$ para amostras independentes. Os dados contínuos sem distribuição normal foram analisados pelo teste U de Mann-Whitney. Os dados qualitativos foram analisados pelo teste do qui-quadrado. 0 teste de correlações lineares de Pearson foi utilizado para as variáveis com distribuição normal, e o coeficiente de correlação de Spearman, para as variáveis sem distribuição normal. A análise de regressão linear múltipla foi realizada nas variáveis independentes que se associaram com a VRT. 0 nível de significância estatístico utilizado foi $p<0,05$.

\section{Resultados}

No período de setembro de 2004 a maio de 2006, 40 dos 41 pacientes acompanhados pelo programa de adultos para FC do HCPA foram incluídos no estudo. Um paciente recusou-se a participar do estudo. Foram estudadas 22 mulheres e 18 homens, sendo a média de idade de $23,7 \pm 6,3$ anos e a média da VRT de $2,5 \pm 0,3 \mathrm{~m} / \mathrm{s}$.

A Tabela 1 resume as características clínicas, funcionais pulmonares, radiológicas e ecocardiográficas dos pacientes de acordo com os valores da VRT. Dezenove pacientes apresentaram valores de VRT $\leq 2,5 \mathrm{~m} / \mathrm{s}$, sendo classificados como sem HP, e 18 pacientes apresentaram valores de VRT $>2,5 \mathrm{~m} / \mathrm{s}$, sendo classificados como tendo HP. Os valores de $\mathrm{VEF}_{1}$ e CVF em \% do previsto foram significativamente menores no grupo com $\mathrm{HP}$ do que no grupo sem HP ( $p<0,001$ e $p=0,001$, respectivamente). Os valores de $\mathrm{SpO}_{2}$ em repouso e no final do teste de caminhada de seis minutos (TC6) foram significativamente menores no grupo com HP do que no grupo sem HP ( $p<0,001$ e $p=0,007$, respectivamente). Os valores de DVD diferiram significativamente entre 
Tabela 1 - Características gerais dos pacientes de acordo com os valores da velocidade de regurgitação tricúspide.

\begin{tabular}{|c|c|c|c|}
\hline Variável & $\begin{array}{c}\text { VRT } \leq 2,5 \mathrm{~m} / \mathrm{s} \\
(\mathrm{n}=19)\end{array}$ & $\begin{array}{c}\text { VRT }>2,5 \mathrm{~m} / \mathrm{s} \\
(\mathrm{n}=18)\end{array}$ & $\mathrm{p}$ \\
\hline ldade (anos) & $22,9 \pm 6,0$ & $25,4 \pm 6,6$ & 0,231 \\
\hline Sexo $(M / F)$ & $9 / 10$ & $9 / 9$ & 0,635 \\
\hline Idade no momento do diagnóstico (anos) & $10(12)$ & $7(21)$ & 0,147 \\
\hline $\mathrm{IMC}\left(\mathrm{kg} / \mathrm{m}^{2}\right)$ & $20,7 \pm 2,9$ & $20,0 \pm 1,6$ & 0,405 \\
\hline Escore clínico & $80(18)$ & $70(16)$ & 0,002 \\
\hline Escore radiológico & $18(6)$ & $15(7)$ & 0,324 \\
\hline $\mathrm{VEF}_{1}$ (\% previsto) & $70,9 \pm 27,7$ & $37,6 \pm 13,9$ & $<0,001$ \\
\hline CVF (\% previsto) & $79,1 \pm 23,8$ & $54,8 \pm 15,8$ & 0,001 \\
\hline $\mathrm{SpO}_{2}$ em repouso $(\%)$ & $97,8 \pm 1,1$ & $95,5 \pm 1,7$ & $<0,001$ \\
\hline $\mathrm{SpO}_{2}$ no final do TC6 $(\%)$ & $96,4 \pm 2,6$ & $90,5 \pm 7,8$ & 0,007 \\
\hline TAS (ms) & $125,3 \pm 34,1$ & $131,2 \pm 19,2$ & 0,519 \\
\hline DVD $(\mathrm{cm})$ & $2,0 \pm 0,4$ & $2,3 \pm 0,3$ & 0,023 \\
\hline DAE $(\mathrm{cm})$ & $3,5 \pm 0,5$ & $3,4 \pm 0,4$ & 0,714 \\
\hline DDVE $(\mathrm{cm})$ & $4,8 \pm 0,3$ & $4,7 \pm 0,4$ & 0,428 \\
\hline DSVE (cm) & $3,0 \pm 0,3$ & $2,9 \pm 0,4$ & 0,859 \\
\hline Fração de ejeção (\%) & $67,0 \pm 4,1$ & $65,9 \pm 6,8$ & 0,544 \\
\hline MVE (g) & $135,7 \pm 38,4$ & $138,7 \pm 42,2$ & 0,825 \\
\hline
\end{tabular}

VRT: velocidade de regurgitação tricúspide; IMC: índice de massa corporal; VEF : volume expiratório forçado no primeiro segundo; CVF: capacidade vital forçada; $\mathrm{SpO}_{2}$ : saturação periférica de oxigênio; TC6: teste de caminhada de seis minutos; TAS: tempo de aceleração sistólica; DVD: diâmetro do ventrículo direito; DAE: diâmetro do átrio esquerdo; DDVE: diâmetro diastólico do ventrículo esquerdo; DSVE: diâmetro sistólico do ventrículo esquerdo; e MVE: massa do ventrículo esquerdo.

os dois grupos $(p=0,023)$. A fração de ejeção do ventrículo esquerdo variou de 54 a $76 \%$ em toda a amostra, não havendo diferença significativa entre os dois grupos. Na análise com um ponto de corte conservador para a VRT, 11 pacientes, de um total de 37 , apresentaram valores de VRT $\geq 2,8 \mathrm{~m} / \mathrm{s}$.

A Figura 1 apresenta a expressão gráfica das correlações da VRT com $\mathrm{SpO}_{2}$, escore clínico, $\mathrm{VEF}_{1}$ e CVF em \% do previsto. A VRT correlacionou-se com a $\mathrm{SpO}_{2}$ em repouso $[\mathrm{r}=-0,70$ $(-0,80 ;-0,50) ; p<0,001]$. Além disso, a VRT correlacionou-se com $\mathrm{SpO}_{2}$ no final do TC6 $[\mathrm{r}=-0,47$ $(-0,68 ;-0,19) ; p=0,003]$, escore clínico $[r=-0,63$ $(-0,79 ;-0,40) ; p<0,001]$, escore radiológico $[r=-0,36(-0,60 ;-0,06) ; p=0,030], V_{E F}$ em litros $[r=-0,55(-0,74 ;-0,29) ; p<0,001]$ e em $\%$ do previsto $[r=-0,61(-0,77 ;-0,37) ; p<0,001]$ e CVF em litros $[r=-0,43(-0,65 ;-0,14) ; p=0,008]$ e em $\%$ do previsto $[r=-0,54(-0,73 ;-0,28) ; p=0,001]$. 0 TAS correlacionou-se com escore clínico $[\mathrm{r}=0,37$ $(0,07 ; 0,61) ; p=0,020], \operatorname{VEF}_{1}[r=0,35(0,04 ; 0,60)$; $p=0,028]$ e CVF $[r=0,34(0,03 ; 0,59) ; p=0,037]$ em $\%$ do previsto. 0 DVD correlacionou-se com $\mathrm{SpO}_{2}$ $[r=-0,44(-0,66 ;-0,15) ; p=0,004]$, escore clínico $[r=-0,38(-0,62 ;-0,08) ; p=0,015]$ e $\mathrm{VEF}_{1}$ em \% do previsto $[r=-0,33(-0,58 ;-0,02) ; p=0,037]$.

$\mathrm{Na}$ análise de regressão linear múltipla (método stepwise), o escore clínico, o $\mathrm{VEF}_{1}$, o escore radiológico e a $\mathrm{SpO}_{2}$ em repouso foram incluídas como variáveis independentes, controladas por sexo e idade. Foi demonstrado que a $\mathrm{SpO}_{2}$ em repouso foi a melhor preditora da VRT e que o seu efeito foi independente da associação entre outras variáveis independentes $(p<0,001)$.

\section{Discussão}

Este trabalho, ao avaliar os pacientes de um programa de adultos para FC ( 40 dos 41 pacientes atendidos pelo programa), independente da gravidade da doença pulmonar e utilizando o ecocardiograma com Doppler como instrumento diagnóstico, contribuiu para evidenciar que a prevalência de HP nessa população foi de $49 \%$ quando analisado um ponto de corte para a VRT de $2,5 \mathrm{~m} / \mathrm{s}$ e de $30 \%$ quando analisado um ponto de corte de VRT de $2,8 \mathrm{~m} / \mathrm{s}$.

Ainda em contraposição aos estudos prévios da literatura ${ }^{(8,9)} \mathrm{em}$ nosso trabalho a VRT foi utilizada 

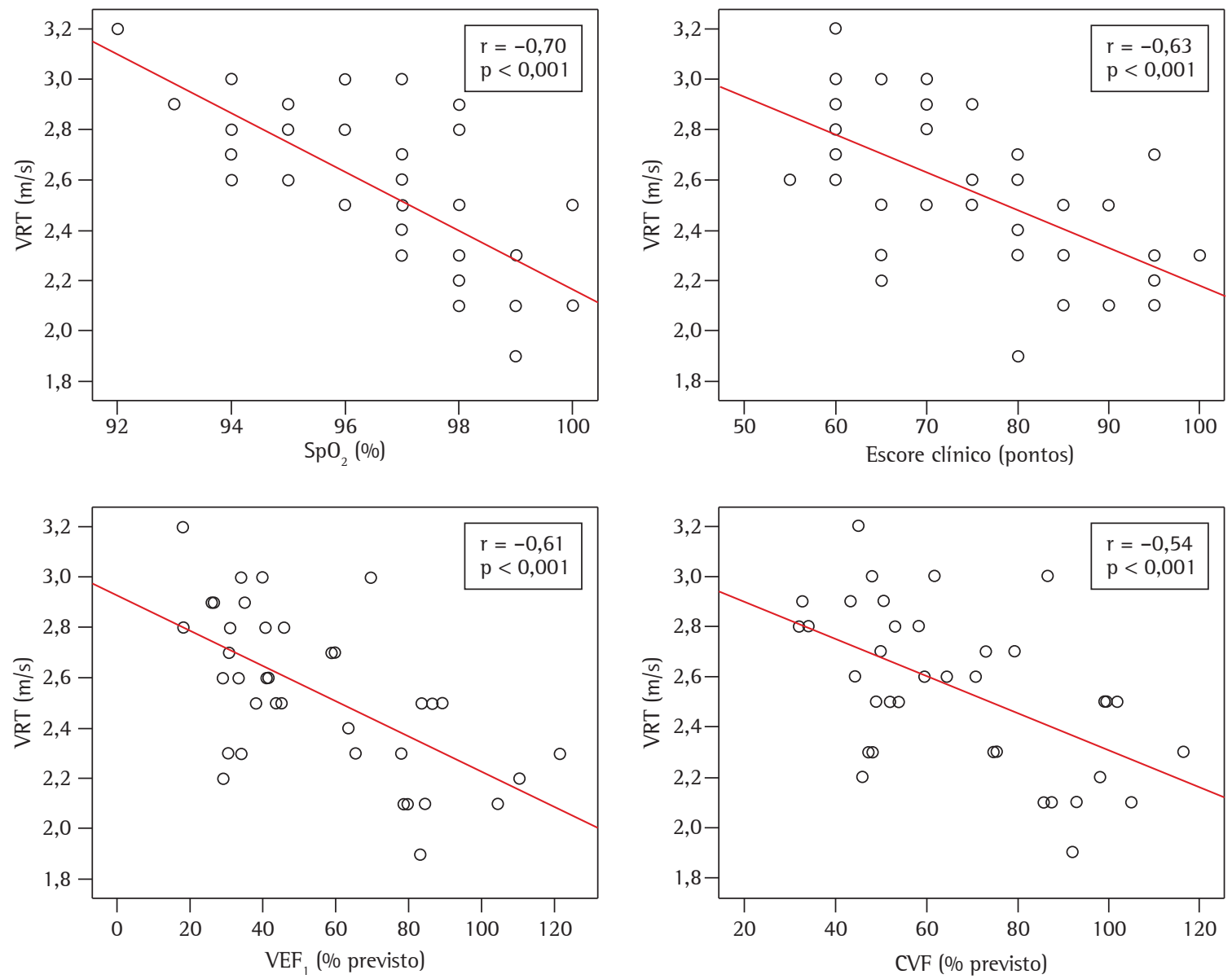

Figura 1 - Correlações da velocidade de regurgitação tricúspide (VRT) com a saturação periférica de oxigênio $\left(\mathrm{SpO}_{2}\right)$, o escore clínico, o volume expiratório forçado no primeiro segundo ( $\left.\mathrm{VEF}_{1}\right)$; e a capacidade vital forçada (CVF).

como variável definidora de HP. Os pacientes classificados como tendo HP apresentaram valores do escore clínico, $\mathrm{VEF}_{1}$, CVF e $\mathrm{SpO}_{2}$ em repouso e no final do TC6 significativamente menores do que os pacientes sem HP. A VRT se correlacionou inversamente com os marcadores de gravidade da doença pulmonar $\left(\mathrm{SpO}_{2}\right.$ em repouso, escore clínico, escore radiológico, $\mathrm{VEF}_{1}$ e CVF), enquanto o TAS e o DVD se correlacionaram de maneira mais fraca com essas variáveis.

0 método ecocardiográfico para a estimativa não-invasiva das pressões em artéria pulmonar baseia-se na medida da VRT. ${ }^{(16)} \mathrm{A}$ VRT reflete a diferença de pressão existente entre o ventrículo direito e o átrio direito, podendo ser calculada pela equação de Bernouille. ${ }^{(12)}$ Quando se adiciona a esse gradiente a estimativa da pressão em átrio direito, obtém-se a pressão sistólica em ventrículo direito. Esse método, simples e facilmente reprodutível, tem demonstrado boa correlação com as medidas invasivas de pressões da artéria pulmonar obtidas em laboratório de hemodinâmica. ${ }^{(17-19)}$

São poucos os estudos que avaliaram HP em pacientes com FC. Os principais são citados a seguir.

Alguns autores ${ }^{(8)}$ estudaram 18 pacientes adultos com FC que apresentavam doença pulmonar grave com o propósito de determinar a prevalência de HP e disfunção cardíaca, estudar as relações entre anormalidades cardiovasculares e hipoxemia e avaliar o impacto da HP subclínica na sobrevida. Observaram HP em 39\% desses pacientes. A pressão sistólica na 
artéria pulmonar (PSAP) se correlacionou significativamente com $\mathrm{VEF}_{1}, \mathrm{SpO}_{2}$ em vigília, $\mathrm{SpO}_{2}$ durante o sono e $\mathrm{SpO}_{2}$ após o exercício. $\mathrm{Na}$ análise multivariada, a $\mathrm{SpO}_{2}$ em vigília foi o maior preditor da PSAP. 0 seguimento clínico dessa coorte por até cinco anos mostrou que a mortalidade foi significativamente maior nos pacientes com HP. Como já foi citado, em nosso estudo foram avaliados todos os pacientes, independentemente da gravidade da doença pulmonar. A prevalência de 49\% de HP identificada em nosso estudo evidencia que mesmo pacientes com doença pulmonar menos grave podem apresentar HP. Da mesma forma que no estudo citado, a regressão linear mostrou que apenas a $\mathrm{SpO}_{2}$ em repouso foi preditiva da PSAP, independentemente de outras variáveis.

Outros autores ${ }^{(9)}$ estudaram três grupos de voluntários: 21 pacientes com FC e doença clinicamente estável (Grupo 1), 5 pacientes com FC e doença muito avançada (Grupo 2) e 23 indivíduos sadios pareados por sexo e idade (Grupo 3). 0 objetivo do trabalho foi avaliar a função ventricular direita utilizando-se o ecocardiograma Doppler tecidual. Observou-se que, nos três grupos, as médias da velocidade sistólica da parede livre do ventrículo direito (VD) foram, respectivamente, $8,9 \pm 1,7 \mathrm{~cm} / \mathrm{s}$, $7,7 \pm 1 \mathrm{~cm} / \mathrm{s}$ e $10,8 \pm 1,9 \mathrm{~cm} / \mathrm{s}(p<0,001)$. As velocidades do ânulo tricúspide foram significativamente menores nos pacientes que nos indivíduos sadios. Os pacientes tiveram um maior tempo de relaxamento isovolumétrico, indicando disfunção diastólica do VD. A espessura da parede do VD foi maior nos pacientes que nos indivíduos sadios ( $p<0,01)$. Concluiu-se que os pacientes com FC apresentavam disfunção subclínica do VD, a qual se correlacionava com a gravidade da doença pulmonar. Em contraposição, nosso estudo não utilizou grupo controle. Também identificamos que a espessura da parede do VD era significativamente maior nos pacientes que apresentaram VRT $>2,5 \mathrm{~m} / \mathrm{s}$, ou seja, no grupo com HP.

Alguns pesquisadores ${ }^{(20)}$ avaliaram, por ecocardiografia, o grau de HP em 45 pacientes com FC (idade entre 3 e 24 anos). A dimensão diastólica final do VD estava aumentada em 67\% dos pacientes, a espessura da parede anterior do VD estava aumentada em 71\% dos pacientes, e a PSAP estava elevada em $84 \%$ dos pacientes. Concluiu-se que o VD sofre importantes alterações com a progressão da doença e que a ecocardiografia pode ser útil na monito- rização clínica evolutiva da gravidade da doença. A prevalência mais elevada de HP, em comparação com nosso estudo, pode decorrer da seleção de pacientes com doença pulmonar mais grave.

Em nosso estudo, a VRT correlacionou-se significativamente com o VEF e a CVF (Figura 1). Os pacientes com VRT $>2,5 \mathrm{~m} / \mathrm{s}$ apresentaram valores significativamente menores de VEF, e CVF. Outros pesquisadores $^{(8)}$ estimaram que mais de $40 \%$ da população de adultos com FC e com doença pulmonar grave tinham HP sem sinais clínicos de cor pulmonale. Pacientes com melhor função pulmonar $\left(\mathrm{VEF}_{1}>40 \%\right.$ do predito) apresentaram menor incidência de HP.

A maior limitação do estudo atual está no método de avaliação da HP. 0 método padrão áureo para diagnóstico de HP é o cateterismo cardíaco e, idealmente, deveria ter sido utilizado. Entretanto, optou-se por realizar a medida pelo método não-invasivo, já que este guarda boa relação com a medida invasiva. ${ }^{(9)} A$ ecocardiografia com Doppler é comumente utilizada para estimar a PSAP e para diagnosticar HP em pacientes com doença pulmonar avançada. Entretanto, devido à grande variabilidade desse método para estimar a PSAP em pacientes com doença pulmonar crônica, alguns trabalhos têm questionado sua utilização para tal fim. ${ }^{(21,22)}$ Alguns autores ${ }^{(21)}$ estudaram uma coorte de 374 pacientes candidatos a transplante pulmonar que foram submetidos a ecocardiografia com Doppler e cateterismo direito para determinar a PSAP e diagnosticar HP. A correlação entre a estimativa da PSAP pela ecocardiografia e a medida pelo cateterismo foi boa $(r=0,69 ; p<0,0001)$. Entretanto, 52\% das estimativas de PSAP foram inacuradas, com mais de $10 \mathrm{mmHg}$ de diferença, quando comparadas com as medidas de PSAP pelo cateterismo, e 48\% dos pacientes foram mal classificados pela ecocardiografia como tendo HP. A sensibilidade, especificidade, valor preditivo positivo e valor preditivo negativo da estimativa da PSAP para o diagnóstico de HP foram, respectivamente, de $85,55,52$ e $87 \%$. Concluiu-se que as estimativas ecocardiográficas de PSAP para o diagnóstico de HP são freqüentemente inacuradas em pacientes com doença pulmonar avançada e levam a superestimar esse diagnóstico. Apesar dessa limitação, a ecocardiografia com Doppler tem a vantagem sobre o cateterismo direito de ser um exame não-invasivo e de mais fácil utilização na prática clínica, especial- 
mente em avaliações repetidas. 0 ponto de corte da VRT para determinação da HP não é bem definido na literatura. A maioria dos estudos tem apresentado um ponto de corte para a VRT $2,5 \mathrm{~m} / \mathrm{s}$ para definir a HP em pacientes com doenças como anemia falciforme. ${ }^{(17,23-25)} 0$ ponto de corte mais conservador para a VRT (de 2,8 m/s) é importante em casos em que não existe confirmação do diagnóstico de HP, pois valores muito baixos de VRT poderiam superestimar a prevalência de HP em certas populações. Além disso, alguns estudos ${ }^{(8,9,23)}$ demonstraram que a identificação de pacientes como tendo HP pela ecocardiografia com Doppler se associou diretamente à pior sobrevida, o que valoriza sua utilização pelo menos como um método de triagem.

Outras limitações do método ficaram expressas, em nosso estudo, quando não foi possível medir o TAS em um paciente, fato atribuído à hiperinsuflação pulmonar. Além disso, em três pacientes a regurgitação tricúspide foi mínima, não passível de aferição pelo método diagnóstico. Esses pacientes não foram incluídos na análise do estudo.

Por fim, o fato de o delineamento do estudo ser transversal não permitiu inferir considerações prognósticas sobre a identificação da HP em nosso grupo de pacientes.

0 presente estudo, realizado em um centro de referência para $\mathrm{FC}$, avaliou a prevalência de HP nos pacientes atendidos pelo programa de adultos para a doença. Ao contrário de trabalhos anteriores, ${ }^{(8,9)}$ onde apenas uma amostra dos pacientes mais graves foi selecionada para inclusão, em nosso estudo foi incluída a grande maioria dos pacientes em acompanhamento, permitindo uma melhor estimativa dessa prevalência. Foi identificada uma prevalência elevada de $\mathrm{HP}$, mesmo em pacientes com $\mathrm{SpO}_{2}$ em repouso ainda normal. Poderia, assim, ser útil monitorar a HP nesses pacientes. A associação entre pior prognóstico e identificação de $\mathrm{HP}$ na $\mathrm{FC}^{(8,9)}$ exige uma melhor avaliação e um acompanhamento mais rigoroso desse grupo de pacientes.

Em conclusão, o presente estudo mostrou que a prevalência de HP nos pacientes acompanhados em um programa de adultos para FC foi de $49 \%$ com VRT > 2,5 m/s e de $30 \%$ considerando-se um ponto de corte conservador para a VRT, sugerindo que a presença de HP seja considerada na avaliação e acompanhamento desses pacientes. 0 grupo classificado como tendo HP apresentou escore clínico, $\mathrm{VEF}_{1}, \mathrm{CVF}$ e $\mathrm{SpO}_{2}$ em repouso significativamente menores que o grupo classificado como sem HP, o que destaca esses parâmetros como preditores de potencial presença de HP nessa população.

\section{Agradecimentos}

Agradecemos a Vânia Naomi Hirakata e a Daniela Benzano a análise estatística. Também agradecemos a todos os membros da equipe do Programa de Adolescentes e Adultos para FC do HCPA a colaboração.

\section{Referências}

1. Ratjen F, Döring G. Cystic fibrosis. Lancet. 2003;361 (9358):681-9.

2. Robinson P. Cystic fibrosis. Thorax.; 56(3):237-41.

3. Camargos PA, Queiroz MV. Pico do fluxo expiratório na avaliação da função pulmonar na fibrose cística. Jornal de Pediatria. 2002;77(1):45-9.

4. Chemla D, Castelain V, Hervé P, Lecarpentier Y, Brimioulle S. Haemodynamic evaluation of pulmonary hypertension. Eur Respir J. 2002;20(5):1314-31.

5. West JB. Doenças ambientais. In: West JB, editor. Fisiopatologia Pulmonar Moderna. 4th ed. São Paulo: Manole; 1996. p. 141-2.

6. Sociedade Brasileira de Pneumologia e Tisiologia. Sociedade Brasileira de Reumatologia. Classificação e avaliação diagnóstica da hipertensão pulmonar. J Bras Pneumol. 2005;31(2):S1-S8.

7. Menna Barreto SS, Gazzana MB. Hipertensão pulmonar: relato de seis casos e atualização do tema. J Pneumol. 2000;26(6):321-36.

8. Fraser KL, Tullis DE, Sasson Z, Hyland RH, Thornley KS, Hanly PJ. Pulmonary hypertension and cardiac function in adult cystic fibrosis: role of hypoxemia. Chest. 1999;115(5):1321-8.

9. lonescu AA, lonescu AA, Payne N, Obieta-Fresnedo l, Fraser $A G$, Shale DJ. Subclinical right ventricular dysfunction in cystic fibrosis. A study using tissue Doppler echocardiography. Am J Respir Crit Care Med. 2001;163(5):1212-8.

10. Gilljam M, Ellis L, Corey M, Zielenski J, Durie P, Tullis DE. Clinical manifestations of cystic fibrosis among patients with diagnosis in adulthood. Chest. 2004;126(4):1215-24.

11. Yankaskas JR, Marshall BC, Sufian B, Simon RH, Rodman D. Cystic fibrosis adult care: consensus conference report. Chest. 2004;125(1 Suppl):1S-39S.

12. Quiñones MA, Otto CM, Stoddard M, Waggoner A, Zoghbi WA; Doppler Quantification Task Force of the Nomenclature and Standards Committee of the American Society of Echocardiography. Recommendations for quantification of Doppler echocardiography: a report from the Doppler Quantification Task Force of the Nomenclature and Standards Committee of the American Society of Echocardiography. J Am Soc Echocardiogr. 2002;15(2):167-84.

13. Shwachman H, Kulczycki LL. Long-term study of one hundred five patients with cystic fibrosis; studies made over a five- to fourteen-year period. AMA J Dis Child. 1958;96(1):6-15. 
14. Brasfield D, Hicks G, Soong S, Tiller RE. The chest roentgenogram in cystic fibrosis: a new scoring system. Pediatrics. 1979;63(1):24-9.

15. Pereira CA, Barreto SP, Simões JG, Pereira FW, Gerstler JG, Nakatani J. Valores de referência para espirometria em uma amostra da população brasileira adulta. J Pneumol. 1992;18(1):10-22.

16. Sbano JC, Tsutsui JM, Terra-Filho M, Mathias Junior W. Papel da ecodopplercardiografia na avaliação da hipertensão pulmonar. J Bras Pneumol. 2004;30(1):78-86.

17. Berger M, Haimowitz A, Van Tosh A, Berdoff RL, Goldberg E. Quantitative assessment of pulmonary hypertension in patients with tricuspid regurgitation using continuous wave Doppler ultrasound. J Am Coll Cardiol. 1985;6(2):359-65.

18. Currie PJ, Seward JB, Chan KL, Fyfe DA, Hagler DJ, Mair $\mathrm{DD}$, et al. Continuous wave Doppler determination of right ventricular pressure: a simultaneous Doppler-catheterization study in 127 patients. J Am Coll Cardiol. 1985;6(4):750-6.

19. Yock PG, Popp RL. Noninvasive estimation of right ventricular systolic pressure by Doppler ultrasound in patients with tricuspid regurgitation. Circulation. 1984;70(4):657-62.

20. Podolska E, Pogorzelski A, Woś H. [Echocardiographic assessment of cor pulmonale in patients with cystic fibrosis] [Article in Polish]. Wiad Lek. 2006;59(3-4):208-13.
21. Arcasoy SM, Christie JD, Ferrari VA, Sutton MS, Zisman DA, Blumenthal NP, et al. Echocardiographic assessment of pulmonary hypertension in patients with advanced lung disease. Am J Respir Crit Care Med. 2003;167(5):735-40.

22. Laaban JP, Diebold B, Zelinski R, Lafay M, Raffoul H, Rochemaure J. Noninvasive estimation of systolic pulmonary artery pressure using Doppler echocardiography in patients with chronic obstructive pulmonary disease. Chest. 1989; 96(6):1258-62.

23. Gladwin MT, Sachdev V, Jison ML, Shizukuda Y, Plehn JF, Minter K, et al. Pulmonary hypertension as a risk factor for death in patients with sickle cell disease. N Engl J Med. 2004;350(9):886-95.

24. Machado RF, Martyr S, Kato GJ, Barst RJ, Anthi A, Robinson MR, et al. Sildenafil therapy in patients with sickle cell disease and pulmonary hypertension. $\mathrm{Br} \mathrm{J}$ Haematol. 2005;130(3):445-53.

25. Machado RF, Anthi A, Steinberg MH, Bonds D, Sachdev $\mathrm{V}$, Kato GJ, et al. N-terminal pro-brain natriuretic peptide levels and risk of death in sickle cell disease. JAMA. 2006;296(3):310-8. 